\title{
elliptique héréditaire je
}

(extraits)

\author{
nathalie stephens
}

This text is marked by the acts of splitting, breaking, undoing, and holds the possibility of reconstitution. elliptique héréditaire je is a mosaic of juxtaposed, decontextualized, enmeshed languages - languages which give echo to uprooted bodies, to languages in danger of extinction, to bodies caught in sexual interstices. Beginning with a line from L'embrasure, "neither one nor the othere, and bothe at once," stephens delves deeply into this paradox, that of the body confronted with its own absence, uncertain geographies, shifting memory, the myth of origins to which it would bind itself.

"Ne néglige pas l'écho; car

c'est d'échos que tu vis."

- Edmond Jabès

\section{je (p)rends ton mal}

dans la chambre bleue tu tends la main de Fatma entre par la fenêtre me convoitant na'bebess et me larmoyante recouvre les yeux fontaine bleue j'avale la mer s'étale dans mon regard vaguant l'âme éc(ar)late une fille s'effiloche

ocre la folie couleur éparpille le vent bleu de mes veines elle court d'eau filigrane

devant rêche la mesquita j'ai douze ans moins que ça ibérique je délasse mes sandales avale une pierre blanche que je sèche dents cassés au pied de la mer Fatma perle sur ma langue cuivrée casbahs effrités Miriam peutêtre ou Kahéna meurs (a)lignée

figure le vent mouvant peine la tracée calomniant ma voix ululée j'avale l'olive m'offre (salée) de l'eau bouche (dé)versée je fúyame trouve sa langue l'écho 


\section{sangre azul}

ha ido la Túria écart(el)ée dígame historique je crie ¡España! mais non Sefarad fumée migratoire mains bleues battant l'eau coulée à mes pieds viens rouge la mer dans l'océan versée noyades au $t^{\prime}$ bila trois fois la pièee mariée ladino ma langue f(i)êlée mosaïque je

ment(h)euse escalade azurée lamentante le mur de berlin hollywood (burl)est-ce que mischling les coudes gamés olvido la croisade topographique la débandade chantons galuth l'argile

je rêve turquoise sur un lit de pierres mains de mes vignobles traversées à Grenade étoile scandée mords Babylone n'existe le passage

labyrinthien Halévi désespère Ibn Ezra la bouche de son amant (dé)figure Andalusía le bleu poème marbré corps arc-boutés drainent el deseo bouches bées la $\mathrm{m}$

erre suffit tu mélopée bégaies sablonneuse ¿quoi España? saph(ir)ique la tentative main posée j'ai mal tes yeux onirique elle m'e veut (réparties)

\section{le secret de la femme}

coulis crépusculaire un livre ouvert je traverse Salomon le désert la bouche posée sur la main paronymique de Fatma te quiero génitale je veux dire oui le livre escríbame la cage torahcique effeuillée goy mon amour la mer bat maghrébine la rive tribu(taire) adónde Kahéna $m^{\prime} \mathrm{e}(\mathrm{n})$ lave cérulée les mains je mamzer

pol(ys)émique tu effleures elle je dis fluviale netilát yadáyim la frontière bois les deux mains nage mémorielle les jambes ouvertes non pas ça fatidique sécrète famulus divines les racines mais

laila féconde rêve la trope errante dis Fatma sororale je judaïque (re)tranchée (mixte) grito le corps captif je te veux isha ma langue dissonante dans les replis de ta vulve la mienne bleutée sacre $\mathrm{l}^{\prime}(\mathrm{a})$ filiation tu $\mathrm{m}^{\prime}$ as 
rêvée nuit bleue à découvert (dé)lyrique la mer dévale mes migrations ma ancestrale

alyatoire le chemin de fer(ule) me déplies tu Marruecos dis-moi l'abjuration de laquelle ma tribu sanguines mes divagations (généillogiques) tu me filament

\section{phosphore}

Oranie dépecée je lis ironique ta main une lignée à la fois dansons éventrées douze fois à la frontière ferrovière (succu)lentes nous $l^{\prime}$ chaim chavirées sais-tu continentale nager? rêvé somnambule la jetée

'crie chair(ie) trompée la langue (en)fourche ici ta main veux-moi sirotant pulpeuse m'avales ent(i)èrement langues et corps figure la toute gouine judaïque fragmentaire (dé)bouchée t'aine la folie

elle court toreador $(\mathrm{e})$

\section{(hy)bride}

furtiva elle esquive pronominale toute appartenance lance énigmatique l'alibi au vent scandant j'étais je t'ai j'ai tes bleu je dis bleue la

s'était voulue maritime pourtant l'écho cogito la terre tekhelet usurpe frangée la mer exige ultramarine l'annulation du trop génétique répond têtue nowhere scand(alis)ant le d'où originaire

elliptique héréditaire j' 